\title{
A Semi-analytic and Experimental Study of Porous Leading Edges
}

\author{
Matthew J. Priddin* and Lorna J. Ayton ${ }^{\dagger}$ \\ University of Cambridge, Cambridge, UK CB3 OWA \\ Paruchuri Chaitanya ${ }^{\ddagger}$ and Philip Joseph ${ }^{\S}$ \\ University of Southampton, Southampton, UK SO17 1BJ
}

\begin{abstract}
This paper presents a semi-analytical investigation into the aeroacoustic properties of aerofoils with porous leading edges, complemented by an experimental study of flat rigid plates with a perforated leading section. Interest in partially porous aerofoils arises from their potential to reduce noise emission from wind turbines and commercial aeroplanes whilst maintaining aerodynamic performance. Developing theoretical models will be helpful for understanding the dominant physical mechanisms and thereby designing optimal implementations. We consider a simple theoretical model that captures acoustic scattering from a finite plate with a porous leading edge. This yields a matrix Wiener-Hopf problem which is solved by an iterative approach that facilitates reliable and rapidly computable solutions. This allows a quantification of acoustic reductions that may be anticipated, and a comparison between studying a porous leading edge on a finite and semi-infinite plate. Experimental results are presented for a flat rigid plate with a perforated leading edge section that demonstrate some qualitative agreement with the theoretical model.
\end{abstract}

\section{Introduction}

Utilizing porosity, permeability and perforations to soften the acoustic properties of a surface is widely known to reduce the far-field noise levels, but is typically detrimental to the aerodynamic performance of the body. This motivates the study of partially porous aerofoils that may provide a satisfactory compromise between aeroacoustic and aerodynamic performance. This paper investigates the use of porous leading edges with view to application in reducing noise emissions by wind turbines and commercial aeroplanes. Mitigating environmental harm due to noise is vital for compliance: meeting new regulatory objectives; and growth: achieving greater capacity and efficiency.

Experimental work by Geyer [1, 2] demonstrates that introducing porosity to an aerofoil can reduce far-field noise at low and mid-range frequencies, but increase high frequency noise, an effect that is likely to be associated with the roughened surface. Concurrent aerodynamic measurements found the intuitive result that porous aerofoils yield higher drag and lower lift. This motivates the consideration of partially porous aerofoils to find optimal configurations. Geyer has investigated porous trailing edges [3], finding improved aerodynamic performance, whilst still attaining noise reductions. Equivalent investigations into porous leading-edges are much less extensive; we note the work of Roger et al. [4] and recent investigations into leading edge perforations on a thick aerofoil [5]. Therefore this paper includes a preliminary experimental investigation of the aeroacoustic performance of porous leading edges on a flat plate for which experimental data is not widely available. Numerical investigations into leading edge porosity include Lee [6], who studied the use of porous leading edge to reduce blade-vortex interaction noise with results indicating substantial reductions are possible, and recent work [7] on wind turbine wakes interacting with a partially porous pylon.

Analytical studies into porosity as a passive noise reduction mechanism have also typically investigated its application to counter trailing edge noise as the dominant contributor to broadband aerofoil self-noise. Jaworski [8] has considered scattering from a semi-infinite poroelastic trailing edge using the model of Howe [9]. This has been extended to consider a finite poroelastic extension to a finite rigid plate without mean flow using Padé approximants [10], and a finite porous extension to a semi-infinite rigid plate [11] using an earlier implementation of the iterative scheme that will be employed here. We also note the numerical investigations of Cavalieri [12, 13] into finite elastic plates. A model for

\footnotetext{
*Graduate Student, Department of Applied Mathematics and Theoretical Physics, Student Member AIAA. mjp98@cam.ac.uk

$\dagger$ EPSRC Early Career Fellow, Department of Applied Mathematics and Theoretical Physics. 1.j.ayton@damtp.cam.ac.uk

${ }^{\ddagger}$ Research fellow, ISVR. C.C.Paruchuri@soton.ac.uk

§Professor of acoustics, ISVR, pfj@soton.ac.uk
} 
the corresponding impact on aerodynamic performance by aerofoils with porosity gradients has also been investigated analytically [14].

Knowing partially porous aerofoils can efficiently reduce trailing-edge noise, we hope to expand their investigation for the reduction of leading-edge noise; $z$ thus we present an investigation into the use of porosity at the leading edge with view to the reduction of gust-aerofoil interaction noise. We consider this problem analytically in order to develop a model suitable for a wide parameter study. We formulate a Wiener-Hopf problem for the scattering of a gust by a finite rigid plate with finite leading edge extension on which an acoustic impedance boundary condition is imposed. This may be associated with porosity or perforations, but neglects hydrodynamic flow alterations and aerofoil geometry. Inclusion of porous-rigid junctions in addition to finite leading and trailing edges leads to $3 \times 3$ (or larger) matrix Wiener-Hopf problems. Whilst similar problems have been approached using Padé approximates [15], this is not ideal as the kernel cannot be uniformly approximated on the real line. In practice this has limited effectiveness to obtaining far-field predictions[10]. We choose to employ the iterative scheme [11, 16] to yield rapid and reliable solutions. The method is discussed and its implementation described in [Paper Control ID: 3086716]. For future studies the speed of our implementation will facilitate near field spatial inversion and integration over a turbulent wavenumber spectra for comparison with experimental results. The experimental results are based on a rigid flat plate where porosity is introduced through a perforated leading edge section, the length of which is varied as a proportion of chord length.

The paper proceeds as follows: we first describe the theoretical and experimental set ups considered. We then present results for the theoretical model and experimental tests. Finally we compare the results and discuss avenues for future study.

\section{Theoretical set up}

This section presents the theoretical modelling of a aerofoil with a porous leading edge.

\section{A. Problem formulation}

We consider an aerofoil of large span in uniform mean flow with velocity $U_{0} \mathbf{e}_{x}$, as presented in figure 1] We model the aerofoil as a finite flat rigid plate of total chord length 1 and infinitesimal thickness. We assume constant density, $\rho_{0}$, and sound speed, $c_{0}$. The porous leading edge is taken to be of length $\zeta$. On the porous and impermeable sections impedance type and no penetration boundary conditions are imposed respectively. We note that the formulation may be easily adapted to consider the simpler case of a finite porous leading edge of length $\zeta$ on a semi-infinite plate, by taking the downstream junction to infinity.

$$
\text { \& incident gust } \phi_{i}
$$
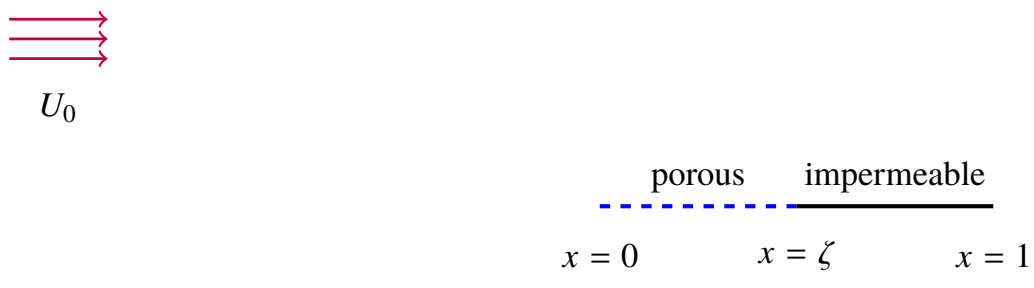

Fig. 1 Schematic diagram of gust-aerofoil interaction considered: harmonic gust incident on a finite rigid plate with finite porous leading section

Governing equation We solve for the total velocity field of the fluid comprising an incident disturbance and a scattered response, each satisifying the convective wave equation. The incident disturbance is taken to be a convective gust of unit strength of the form

$$
g_{i}(x, y) e^{-i \omega t+i k_{3} z}=e^{-i \omega t+i\left(k_{1} x+k_{2} y+k_{3} z\right)}
$$

with $k_{1}=\omega / M c_{0}$ in order to convect with the mean flow. We write the scattered field as $g(x, y) e^{-i \omega t+i k_{3} z}$ and non-dimensionalize velocities with free stream velocity $U_{0}$, corresponding to Mach number $M=U_{0} / c_{0}$. The scattered 
field must satisfy the convective Helmholtz equation

$$
\left[\beta^{2} \frac{\partial^{2}}{\partial x^{2}}+\frac{\partial^{2}}{\partial y^{2}}+2 i \frac{\omega}{c_{0}} M \frac{\partial}{\partial x}+\frac{\omega^{2}}{c_{0}^{2}}-k_{3}^{2}\right] g=0
$$

where $\beta^{2}=1-M^{2}$. Applying a convective transform $h(x, y)=g(x, y) e^{i \omega M x / \beta^{2} c_{0}}$ yields

$$
\left[\beta^{2} \frac{\partial^{2}}{\partial x^{2}}+\frac{\partial^{2}}{\partial y^{2}}+w^{2}\right] h=0
$$

where

$$
w=\frac{1}{\beta^{2}} \sqrt{\frac{\omega^{2}}{c_{0}^{2}}-k_{3}^{2}}
$$

is the wavenumber corresponding to the temporal transform, accounting for convective effects. Now applying the Prandtl-Glauert transformation, the field $\phi(x, y)=h(x / \beta, y)$ satisfies the Helmholtz equation

$$
\left[\frac{\partial^{2}}{\partial x^{2}}+\frac{\partial^{2}}{\partial y^{2}}+w^{2}\right] \phi=0
$$

Boundary conditions On the porous leading edge an impedance type boundary condition is imposed as in [11] with porosity described by a parameter $\mu$, and on the impermeable section the normal particle velocity must be zero. Away from the aerofoil we require continuity of pressure across $y=0$ and we require continuity of particle displacement everywhere. These yield boundary conditions for $\phi$

$$
\begin{aligned}
& {[\phi(x, 0)]_{-}^{+}=0 \quad \text { upstream }-\infty<x<0} \\
& \phi^{\prime}(x, 0)-\frac{\mu}{2}[\phi(x, 0)]_{-}^{+}=q_{2}(x) \quad \text { porous } \quad 0<x<\zeta \\
& \phi^{\prime}(x, 0)=q_{3}(x) \text { impermeable } \quad \zeta<x<1 \\
& {[\phi(x, 0)]_{-}^{+}=\quad 0 \quad \text { downstream } \quad 1<x}
\end{aligned}
$$

where we have written $\frac{\partial \phi}{\partial y}=\phi^{\prime}$ and $[\phi(x, 0)]_{+}^{-}$denotes the jump in $\phi(x, y)$ across $y=0$.

Integral transforms We define the $x$-Fourier transform for a function $\phi(x, \cdot)$ by

$$
\Phi(\alpha, \cdot):=\int_{-\infty}^{\infty} \phi(x, \cdot) e^{i \alpha x} \mathrm{~d} x
$$

and partial range transforms

$$
\begin{aligned}
\Phi_{[-, L]}(\alpha, \cdot) & :=\int_{-\infty}^{L} \phi(x, \cdot) e^{i \alpha x} \mathrm{~d} x \\
\Phi_{[L,+]}(\alpha, \cdot) & :=\int_{L}^{\infty} \phi(x, \cdot) e^{i \alpha x} \mathrm{~d} x \\
\Phi_{\left[L_{1}, L_{2}\right]}(\alpha, \cdot) & :=\int_{L_{1}}^{L_{2}} \phi(x, \cdot) e^{i \alpha x} \mathrm{~d} x
\end{aligned}
$$

Fourier transforming equation 4 in $x$ find general solution decaying as $|y| \rightarrow \infty$ is given by

$$
\Phi(\alpha, y)= \begin{cases}A(\alpha) e^{-y \gamma(\alpha)} & y>0 \\ B(\alpha) e^{+y \gamma(\alpha)} & y<0\end{cases}
$$

where $\gamma(\alpha)=\sqrt{\alpha^{2}-w^{2}}$ with branch cuts chosen to be the rays $\pm w+e^{ \pm i \pi / 2}[0, \infty]$. Imposing continuity of particle normal velocity requires $\Phi$ to be anti-symmetric in $y$, that is $A=-B$. Restricting attention to $y>0$ the boundary 
conditions on $\Phi=\Phi(\alpha, 0+)$ become

$$
\begin{aligned}
\Phi_{[-, 0]} & =0 \\
\Phi_{[0, \zeta]}^{\prime}-\mu \Phi_{[0, \zeta]} & =Q_{2} \\
\Phi_{[\zeta, 1]}^{\prime} & =Q_{3} \\
\Phi_{[1,+]} & =0
\end{aligned}
$$

Explicitly, for an incident harmonic disturbance with streamwise wavenumber $\delta$ (in the transformed domain) incident upon a section $\left[\xi_{\text {start }}, \xi_{\text {end }}\right]$ of the plate chord, the forcing terms are proportional to

$$
\begin{aligned}
Q_{2} & =-\frac{e^{i(\alpha+\delta) \zeta}-e^{i(\alpha+\delta) \xi_{\text {start }}}}{\alpha+\delta} \\
Q_{3} & =-\frac{e^{i(\alpha+\delta) \xi_{\text {end }}}-e^{i(\alpha+\delta) \zeta}}{\alpha+\delta}
\end{aligned}
$$

where $\delta=k M / \beta$.

Wiener-Hopf Problem Substituting the boundary conditions into the relationship $\Phi^{\prime}+\gamma \Phi=0$ we find the spectral equation

$$
(\gamma+\mu) \Phi_{[0, \zeta]}+\gamma \Phi_{[\zeta, 1]}+\Phi_{[-, 0]}^{\prime}+Q_{2}+Q_{3}+\Phi_{[1,+]}^{\prime}=0
$$

If we a priori knew two of the unknowns such that only an adjacent pair in the listing $\Phi_{[-, 0]}^{\prime}, \Phi_{[0, \zeta]}, \Phi_{[\zeta, 1]}, \Phi_{[1,+]}^{\prime}$ remained unknown then this would form a scalar Wiener-Hopf equation. These correspond to solving a Wiener-Hopf problem associated with each physical junction:

$$
\begin{aligned}
\Phi_{[-, 0]}^{\prime}+(\gamma+\mu) \Phi_{[0, \zeta]} & =-Q_{2}-Q_{3}-\gamma \Phi_{[\zeta, 1]}-\Phi_{[1,+]}^{\prime} \\
(\gamma+\mu) \Phi_{[0, \zeta]}+\gamma \Phi_{[\zeta, 1]} & =-Q_{2}-Q_{3}-\Phi_{[-, 0]}^{\prime}-\Phi_{[1,+]}^{\prime} \\
\gamma \Phi_{[\zeta, 1]}+\Phi_{[1,+]}^{\prime} & =-Q_{2}-Q_{3}-\Phi_{[-, 0]}^{\prime}-(\gamma+\mu) \Phi_{[0, \zeta]}
\end{aligned}
$$

Each equation must be scaled by an exponential factor corresponding to the junction location in order to apply Liouville's theorem when carrying out the Wiener-Hopf method.

The corresponding system for a semi-infinite plate arises similarly, in this case from the spectral equation

$$
(\gamma+\mu) \Phi_{[0, \zeta]}+\gamma \Phi_{[\zeta,+]}+\Phi_{[-, 0]}^{\prime}+Q_{2}+Q_{3}=0
$$

\section{B. Iterative Wiener-Hopf approach}

The formulation in equation 12 enables us to construct a fixed-point iterative scheme by using previously computed values for the forcing on the left hand side of equation 12 and taking an initial guess for certain unknown functions. This is motivated by associating these "coupling" terms with backscattering, which we suppose on physical grounds to be weak. We initially take unknown terms to be zero and solve the equations corresponding to junctions at $x=0$, then $\zeta$, then 1 , then $\zeta$ and repeat.

The iterative approach and numerical implementation employed is presented and discussed in [Paper Control ID: 3086716]. It is coded in Julia and utilizes the SingularInTEGRALEQUATIONS.JL package[17]. We use a numerical factorization of the scalar kernel $\gamma+\mu$ following a similar methodology to [18].

Spatial inversion The solution $\phi$ may be recovered by inverting the spatial Fourier transform:

$$
\phi(x, y)=\frac{\operatorname{sgn} y}{2 \pi} \int_{-\infty}^{\infty} \Phi(\alpha, y) e^{-|y| \gamma(\alpha)-i \alpha x} \mathrm{~d} \alpha
$$

To obtain the acoustic pressure, we use the relation

$$
p(x, y)=-\left(\frac{\partial \phi}{\partial x}-\frac{i k_{1}}{\beta^{2}} \phi\right) e^{-i k_{1} M^{2} x / \beta^{2}}
$$

The far-field may be approximated by the method of steepest descent. Of particular interest is the far-field directivity $D(\theta)$, defined in [11] as

$$
\phi(r, \theta) \sim D(\theta) \frac{e^{i w r}}{\sqrt{r}}, \quad r \rightarrow \infty
$$




\section{Modelling}

We now review some of the key modelling assumptions.

Porosity To consider a softer acoustic boundary condition, it is natural mathematically to impose a Robin boundary condition for the velocity potential. It is less clear how the associated parameter $\mu$ relates to a physical example of a porous material. Based on simplified modelling in [9] previous theoretical works [8, 11] have considered taking

$$
\mu=\frac{\alpha_{H} K_{R}}{\pi R^{2} \beta}
$$

for a porous plate with evenly spaced circular apertures of radius $R$, Rayleigh conductivity $K_{R}=2 R$ and fractional open area $\alpha_{H}$. For the model to be valid we require small fractional area $\alpha_{H} \ll 1$ and the length scale of the incident disturbance to be much larger than the aperture radius, $k_{0} R \ll 1$.

We note that the associated derivation does not model viscous effects, and the value for the Rayleigh conductivity $K_{R}=2 R$ is strictly derived for the case of no mean flow. An improved model may be developed based upon further examples in [9]. Viscous dissipation may be included by modelling vortex shedding, and yields a value of $\mu$ with non-zero imaginary part and that is frequency dependent, as when modelling impedance liners [9, 19]. The theoretical scattering model presented may be readily applied for a more sophisticated, perhaps frequency dependent, porosity parameter.

Incident disturbance The present work seeks to assess the role of gust-aerofoil interaction noise in the presence of a porous leading edge. Our theoretical model allows us to consider arbitrary harmonic incident disturbances. To further interrogate the models we will consider the gust to force varying sections of the rigid plate. This is motivated in part by a desire to avoid spurious results from the incident gust interacting strongly with the trailing edge since here the turbulent boundary layer is anticipated to strongly affect results. We instead hope to only capture backscattering effects. In the experimental work the boundary layer is tripped shortly after the porous section, so we typically suppose the gust is incident only on the first $30 \%$ of a finite chord.

\section{Experimental set up}

\section{A. Open-jet test facility and instrumentation}

Far-field noise measurements have been carried out at the Institute of Sound and Vibration Research's open-jet wind tunnel facility. The wind tunnel is located within the anechoic chamber, of dimension $8 \mathrm{~m} \times 8 \mathrm{~m} \times 8 \mathrm{~m}$ as shown in figure 2. The walls are acoustically treated with glass wool wedges and the cut-off frequency is $80 \mathrm{~Hz}$. The nozzle has dimensions of $150 \mathrm{~mm}$ and $450 \mathrm{~mm}$ and provides a maximum flow speed of $100 \mathrm{~m} / \mathrm{s}$. A detailed description of the wind tunnel, including its characteristics, is presented by [20]. To maintain two-dimensional flow around the aerofoil, side plates are mounted to the nozzle exits which will also support the aerofoil in the flow. The mean leading edge of the aerofoil is located $150 \mathrm{~mm}$ downstream from the nozzle exit.

In order to prevent tonal noise generation due to Tollmien-Schlichting waves convecting in the laminar boundary layer, and to ensure complete consistency between the different cases, the flow near the leading edge of the aerofoil was tripped to force transition to turbulence using a rough band of tape of width $1.25 \mathrm{~cm}$ located $33 \%$ of chord from the leading edge, on both suction and pressure sides. The tape has roughness of SS 100, corresponding to a surface roughness of $140 \mu \mathrm{m}$. Transition is forced by the use of the trip tape, which is many orders of magnitude rougher than the aerofoil surface, and is therefore highly unlikely to affect transition. Previous noise measurements in our facility have indicated that self-noise is insensitive to the method of tripping.

\section{B. Far-field noise measurements}

Far-field noise measurements from the flat plate were made using 11, half-inch condenser microphones (B\&K type 4189) located at a constant radial distance of $1.2 \mathrm{~m}$ from the mid span of the flat plate leading edge. These microphones are placed at emission angles of between $40^{\circ}$ and $140^{\circ}$ measured relative to the downstream jet axis. Measurements were carried for $10 \mathrm{~s}$ duration at a sampling frequency of $50 \mathrm{kHz}$, and the noise spectra was calculated with a window 


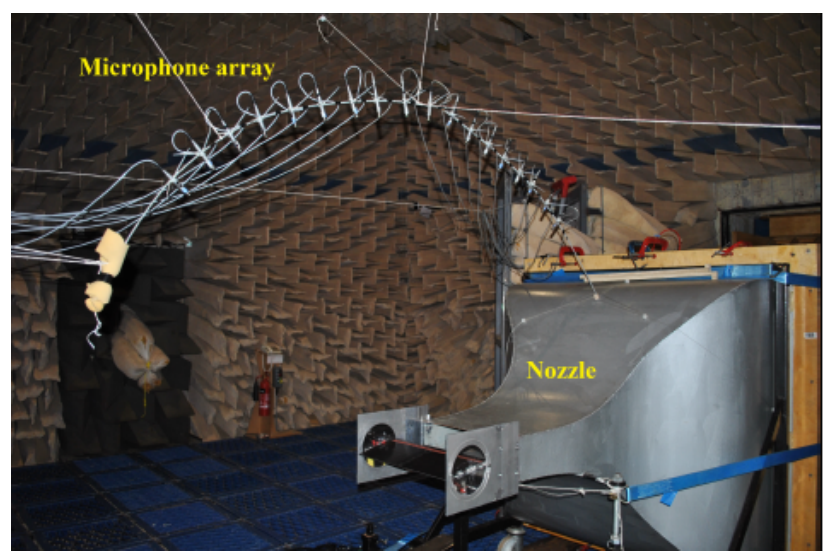

Fig. 2 Photograph of jet nozzle and test setup inside the ISVR anechoic chamber.

size of 1024 data points corresponding to a frequency resolution of $48.83 \mathrm{~Hz}$ and a $B T$ product of about 500 , which is sufficient to ensure negligible variance in the spectral estimate at this frequency resolution.

The acoustic pressure at the microphone was recorded at the mean flow velocities $(U)$ of 20,40 and $60 \mathrm{~m} / \mathrm{s}$. Noise reductions are presented in terms of the Sound Power Level spectra $\operatorname{PWL}(f)$ calculated by integrating the pressure spectra over the polar array of 11 microphones using the procedure described in [21]. Sound power level reductions are determined by subtracting the sound power level spectra due to the porous aerofoil configuration from that due to the baseline impermeable profile.

\section{Turbulence characterisation}

A bi-planar rectangular grid with overall dimensions of $630 \times 690 \mathrm{~mm}^{2}$ located in the contraction section $75 \mathrm{~cm}$ upstream of the nozzle exit was used to generate turbulent flow that provides a velocity spectrum that is a close approximation to homogeneous and isotropic turbulence at the aerofoil leading edge. However, we emphasize that the condition of isotropy is not a key requirement for predicting the noise radiation but only that the velocity spectrum at the aerofoil leading edge is known. A comparison of the streamwise velocity spectra measured at $145 \mathrm{~mm}$ from the nozzle exit $\left(S_{u u} / U\right)$ plotted against $f / U$ is compared in figure 3 to the theoretical Liepmann velocity spectrum, where the mean square velocity and integral length scale are chosen to give best fit to the measured data. Close agreement is observed for $2.5 \%$ turbulence intensity and a $7.5 \mathrm{~mm}$ streamwise integral length-scale.

\section{Porous insert manufacture}

A parametric experimental study was undertaken to investigate the effect on radiated noise due to variations in porous length on rigid flat plates in-accordance to the analytic model presented in section $\Pi$. The rigid flat plate is constructed from two metallic plates of $1 \mathrm{~mm}$ thickness riveted together. The flat plate porous section made from metal plate of $1.6 \mathrm{~mm}$ thickness are inserted in between the two $1 \mathrm{~mm}$ plates. The two steps caused by the porous inserts are ground down to smooth the step. The trailing edge of the plate is sharpened to prevent vortex shedding noise, although the leading edge was left blunt i.e. was not sharpened like the trailing edge for consistency across each porous geometry investigated. The dimensions of the flat plate are $L_{0}=15 \mathrm{~cm}$ mean chord and $S=45 \mathrm{~cm}$ span respectively. More details about the flat plate main body is given in [21].

A schematic of the porous geometry is shown in figure 4 , where the modified flat plate is located in a turbulent flow of velocity $U$. The principle dimensions are shown in figure 4 , where $R$ is the radius of the hole, $T$ is the distance between the hole. In the current study we consider a fixed porosity of $32.8 \%$ and the perforation dimensions are $R=1.5$ $\mathrm{mm}$ and $T=5 \mathrm{~mm}$ respectively. To validate our analytic model presented in section III measurements are conducted on three different porous sections of $6 \%, 14.7 \%$ and $26.7 \%$ of aerofoil chord for a fixed porosity of $32.8 \%$. The number of porous hole rows open for the each case are 2, 5 and 9 respectively. A baseline (impermeable leading edge) is also considered for reference. 


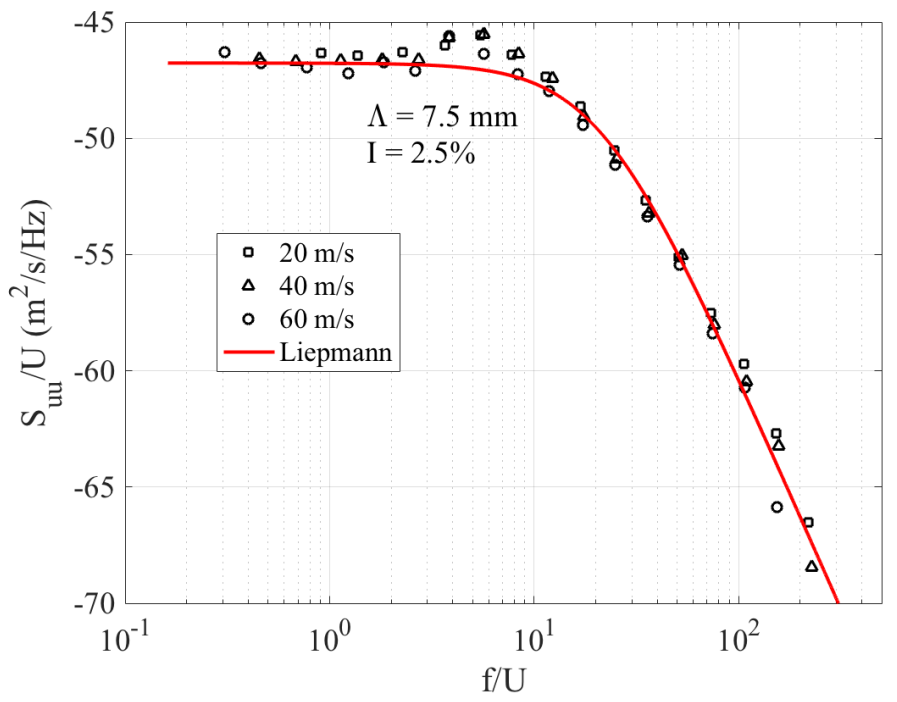

Fig. 3 Comparison between the measured axial velocity spectra and theoretical Liepmann spectra.

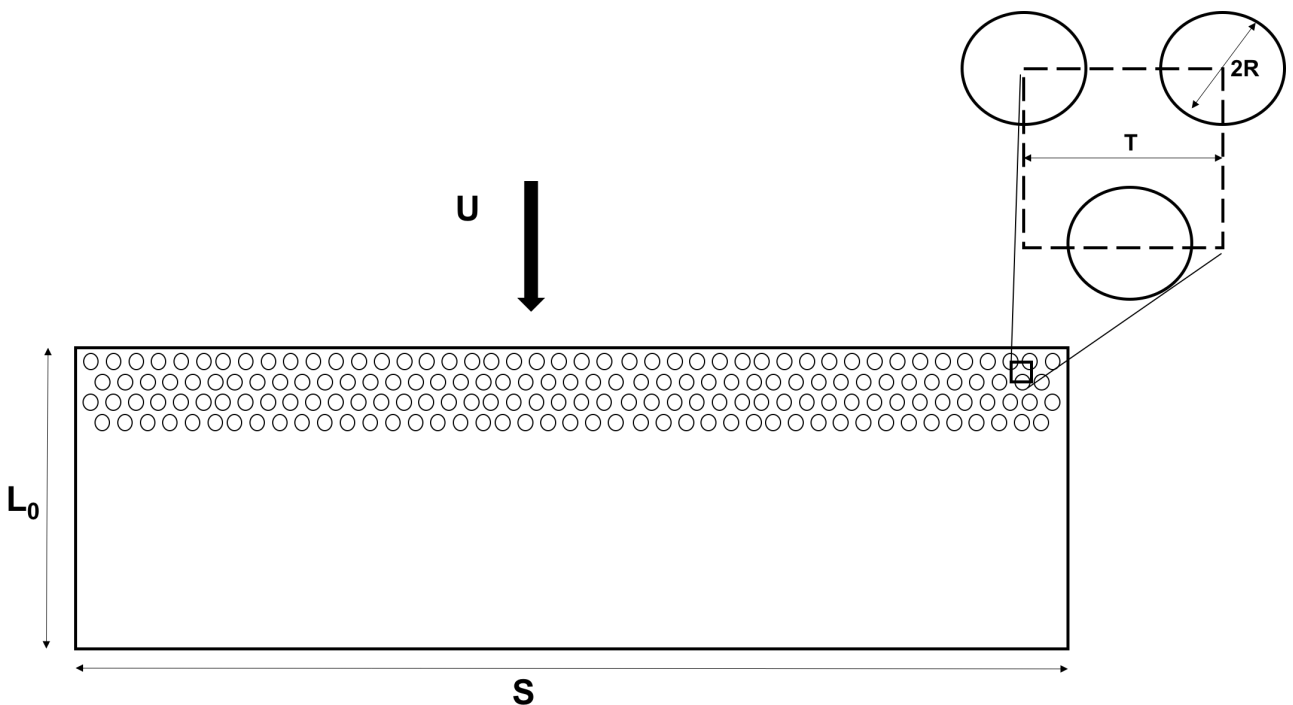

Fig. 4 Schematic of porous leading edge with dimensions.

\section{Results}

In this section we present results demonstrating the noise reductions obtained by porous leading edge.

\section{A. Theoretical aeroacoustic results}

Our theoretical model allows us to investigate the role of the length of the porous leading edge as a proportion of the chord $\zeta$ and porosity of this section $\mu$ for alternative incident fields (gust frequency $k_{1}, k_{3}$ and Mach number $M$ of the background flow, and where on the plate this disturbance is incident). We will typically consider only the dominant spanwise wavenumber $k_{3}=0$, and consider a dimensional lengthscale based upon the chord of the experimental aerofoil, $L_{0}=150 \mathrm{~mm}$.

Validation We first note that when the porous extension forms a small (1\%) and large (99\%) proportion of the chord the directivity patterns collapse onto those for a fully rigid and fully porous plate respectively, as may be observed in 
figure 5 The iterative approach for a finite plate has been validated against the independent Mathieu function solution for scattering from a finite rigid plate in [Paper Control ID: 3086716], which is a fully analytical solution.

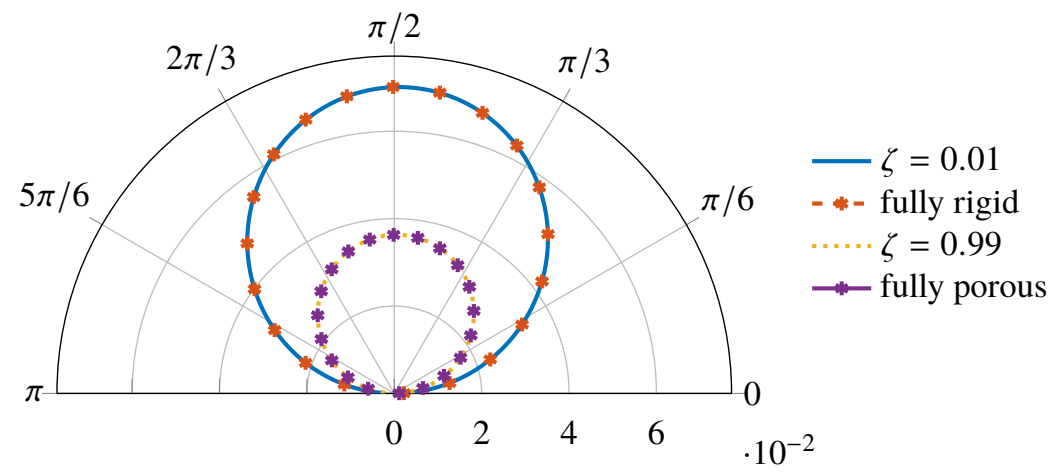

Fig. 5 Validation cases: directivity patterns porous extension to $0 \%$ and $100 \%$ chord with $\mu=2$ for a convected gust associated with $\omega L_{0} / c_{0}=1$ and $M=0.5$. For extreme values answers agree up to approximately the tolerance imposed on the numerical scheme.

Directivity patterns Figure 6 6 presents example directivity patterns for a gust in mean flow of Mach number $M=0.3$ and wavenumber $k=\omega L_{0} / c_{0}=8$. Panel (a) shows that as we increase $\zeta$ (the length of the porous leading edge) we typically find a reduction in far-field noise level, tending towards the directivity pattern associated with a fully porous plate. However, the introduction of the porous junction at certain lengths $(\zeta=0.2)$ can lead to notable increase in noise due to constructive interference between the two scattered acoustics fields (the leading edge, and the junction). Panel (b) shows that increasing the porosity $\mu$ for a fixed length of leading edge $\zeta=0.3$ tends to decrease the far-field noise. We note that substantial reductions become significant when $\mu / k$ is $O(1)$. In all these cases the introduction of a porous leading edge leads to a shift in the directivity pattern towards the downstream direction.

Overall noise level and turbulent wavenumber spectrum We now consider an overall measure of the far-field directivity level by integrating over angles from $[0.2 \pi, 0.8 \pi]$, approximately the arc $40^{\circ}$ to $140^{\circ}$ over which the experimental microphone array is placed.

To find an overall sound pressure level (SPL) prediction we integrate the semi-analytical far-field spectrum over a Liepmann spectra, as in Ayton \& Chaitanya [22]. The theoretical SPL prediction is computed as

$$
10 \log _{10}\left(\int_{-\infty}^{\infty}\left|p^{*}\left(r, \theta, k_{1}, k_{3}\right)\right|^{2} \Pi^{(\infty)}\left(k_{1}, k_{3}\right) \mathrm{d} k_{3}\right)
$$

where $\Pi^{(\infty)}\left(k_{1}, k_{3}\right)$ is the upstream Liepmann spectrum given by

$$
\Pi^{(\infty)}\left(k_{1}, k_{3}\right)=\frac{3\left(\overline{u^{*}} \Lambda^{*}\right)^{2}}{4 \pi} \frac{\Lambda^{2}\left(k_{1}^{2}+k_{3}^{2}\right)}{\left(1+\Lambda^{2}\left(k_{1}^{2}+k_{3}^{2}\right)\right)^{5 / 2}}
$$

where $\Lambda=0.05$ is the non-dimensional integral lengthscale of turbulence (defined as the integral length scale, $7.5 \mathrm{~mm}$, divided by the chord length $150 \mathrm{~mm}, \Lambda^{*}=0.0075 \mathrm{~m}$ the dimensional integral length scale, and $\overline{u^{*}}=0.025 U^{*}$ is the turbulence intensity ( $2.5 \%$ of the free stream velocity, $\left.U^{*}\right)$. In the expression for the SPL $p^{*}$ denotes the dimensional pressure, $p^{*}=\rho_{0}^{*}\left(U^{*}\right)^{2} p(r, \theta)$, where $p$ is the far-field pressure given analytically and $\rho_{0}^{*}=1.1225 \mathrm{kgm}^{-3}$. For a given frequency disturbance we would restrict $k_{3}$ values to $\left|k_{3}\right|<k_{1}$ as is expected in the experimentally generated grid-turbulence, and further so as to only consider propagating modes. In this paper results are presented for $k_{3}=0$, the dominant spanwise wavenumber, and present total SPL levels in arbitrary units to demonstrate relative trends in oscillations observed.

Frequency dependence We now consider how the reductions occur at different frequencies, and further how this is related to the introduction of different lengthscales. Figure 7 presents reduction for a fixed porosity $\mu=2$ for a in 


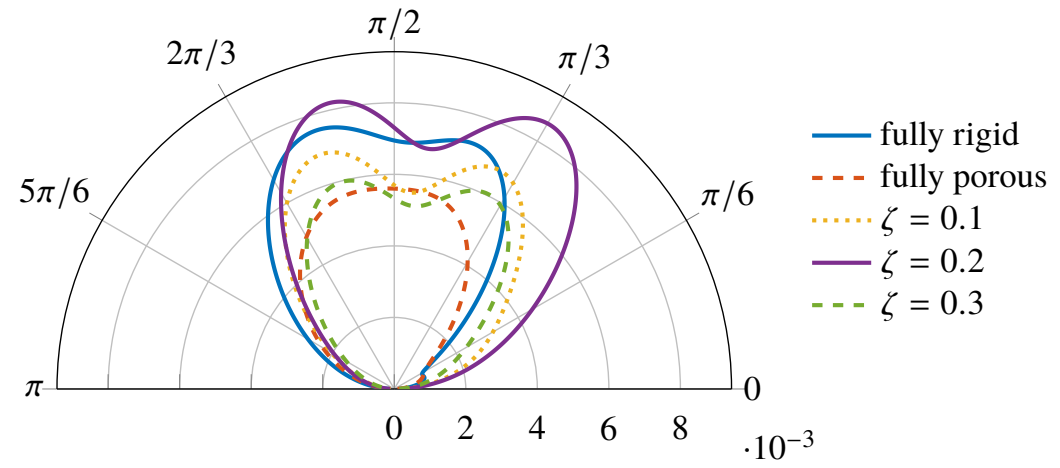

(a) Far-field directivity varying length of a porous leading edge of porosity $\mu=5$

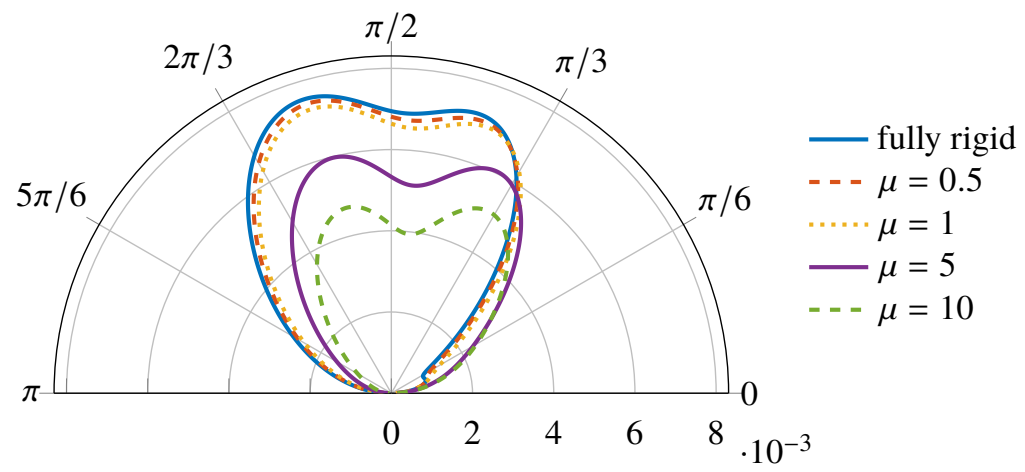

(b) Far-field directivity varying porosity $\mu$ of an leading edge $30 \%$ of chord

Fig. 6 Scattering of a plane wave from a finite rigid plate with a porous leading edge. Gust $M=0.3$ and $k=\omega L_{0} / c_{0}=8$ 
mean flow $U_{0}=40 \mathrm{~m} / \mathrm{s}$. We first consider a semi-infinite plate with a porous leading edge of length $\zeta L_{0}$. Results for a convected gust are presented in panel (a) - change in SPL and (b) - total SPL. In (b) we see how introducing the lengthscale of the porous section adds oscillations in the SPL compared to a rigid half-plane. These correspond to sound reductions and inevitably collapse when rescaling by the length of porous section as in (a), with the largest reduction occurring at Strouhal number $f \zeta L_{0} / U_{0} \approx 0.5$, and a series of smaller reductions at higher frequencies.

We now consider the effect of finite chord. with results presented in panels (c) and (d). In panel (c) we consider a gust incident on the first $30 \%$ of the chord, as after this point the boundary layer is tripped in the experimental set up, and so leading and trailing edge incident spectra might be anticipated to become uncorrelated. In panel (d) we present the baseline of a gust incident on the entire chord. In this case we find strong constructive and destructive interference associated with the lengthscale of the chord, with increases occurring as strongly localized spikes around these critical frequencies, and wider band reductions skewed towards lower frequencies. Forcing only a finite section reduces these spikes, and so we find more uniform reductions. We find larger reductions at lower frequencies by comparison with the semi-infinite plate. This might be expected as the chord may be considered to be increasingly compact.

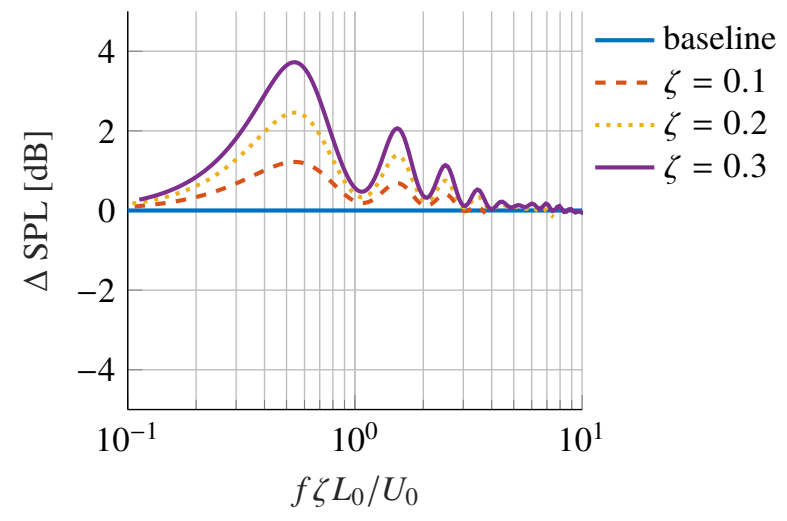

(a) Reduction - semi-infinite plate

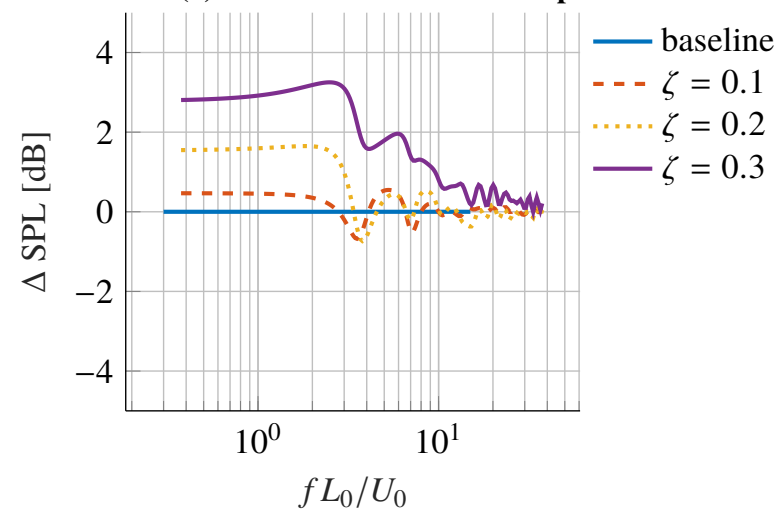

(c) Reduction - finite plate forcing leading $30 \%$ chord, $[x \in$ $[0,0.3]$

Fig. 7 Far-field noise level integrated over directivities $\theta \in[0.2 \pi, 0.8 \pi]$ for $\mu=2, L_{0}=0.15, U_{0}=40$ varying the porous leading extension as a proportion $\zeta$ of the chord. Note the overall SPL level is in arbitrary units, highlighting the relative oscillations of the profiles.

Porosity dependence Figure 8 presents the typical effect of varying porosity of a leading edge of length $\zeta$ on a finite plate for a frequency at which a reduction occurs, $\omega L_{0} / c_{0}=1$ and $M=0.2$. Whilst the reduction typically increases with increased porosity, this benefit diminishes as the porosity is increased further. 


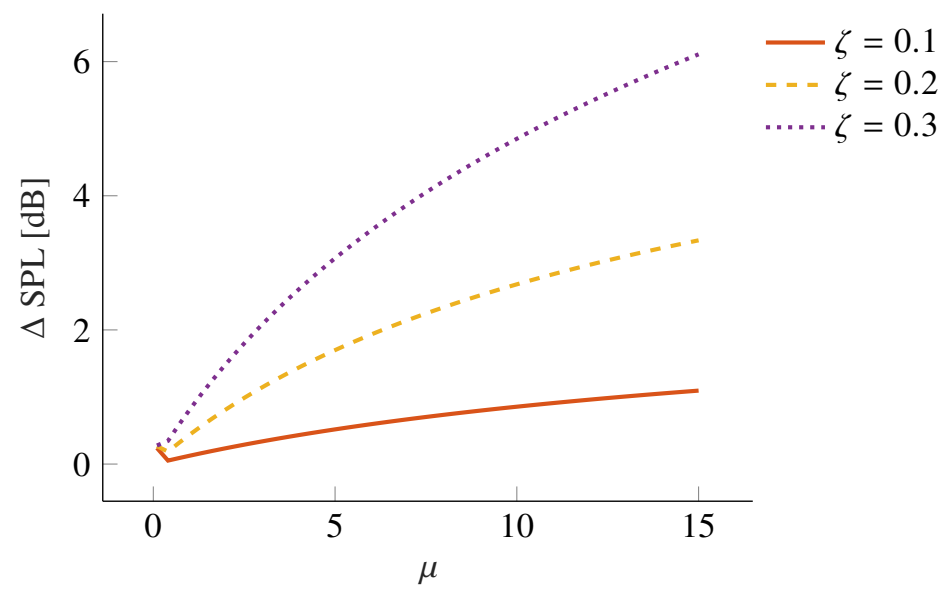

Fig. 8 Far-field sound reduction for a finite plate with porous leading edge, varying porosity for $\omega L_{0} / U_{0}=1$ and $M=0.2$

\section{B. Experimental results}

We first demonstrate the typical sound power spectra radiated from a baseline and porous leading edge in a turbulent flow. The total noise radiated by the aerofoil located within a turbulent flow is dominated by leading-edge noise at the chosen frequencies, and by trailing-edge noise at higher frequencies. As it is very difficult to measure leading-edge noise and trailing-edge self-noise separately, our experimental analysis was limited to the frequency region where leading-edge interaction noise dominates trailing-edge self-noise.

Figure 9 shows a typical total sound power spectra and self-noise spectra for a porous leading edge of length $\zeta=0.267$ with $32.8 \%$ porosity along with the total and self-noise of baseline aerofoil. The spectra of radiated noise due to trailing-edge self-noise alone is obtained by removing the turbulence grid. We observe an increase in noise due to the porous leading edge both in total and self-noise spectra. This clearly demonstrates that the increase in total radiated noise is due to the self-noise; this is due to an increase in cross-flow across the flat plates making the flow more turbulent which in turn increases the noise. The frequency at which this increase occurs is mostly likely function of porosity and hole diameter. More work is required to shows the influence of hole diameter and porosity on noise increase. This paper now focuses on considering different opening length and velocities in order to obtain significant noise reductions compared to the baseline aerofoil.

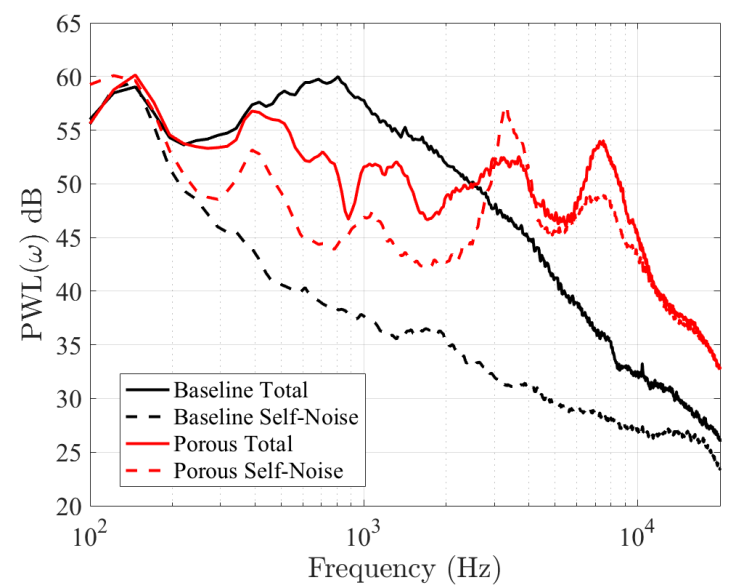

Fig. 9 Comparison of noise spectra for baseline and porous flat plate of $32.8 \%$ porosity and $\zeta=0.267$ at $U_{0}=60 \mathrm{~m} / \mathrm{s}$

Total radiated sound power spectra and sound power reductions are plotted in Figure 10 against frequency for various 
open section length of $\zeta=0.06,0.147,0.267$ respectively for $32.8 \%$ open area ratio and flow velocities of 40 and $60 \mathrm{~m} / \mathrm{s}$. Significant noise reductions are observed for two largest values of $\zeta=0.147$ and 0.267 in the mid-frequency region $(<2500 \mathrm{~Hz})$. The increase in noise after $2500 \mathrm{~Hz}$ is due to flow noise generated as described above. With the increase of $\zeta$ we observe the noise reductions move towards low frequencies. The reason for negligible noise reductions in the case $\zeta=0.06$ might be due to masking of the self-noise which is due to cross-flow across the flat plate.

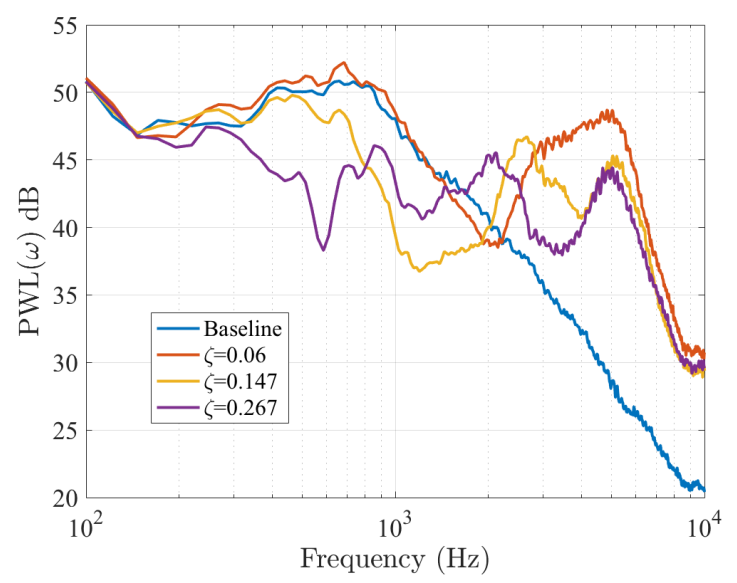

(a) $U=\mathbf{4 0} \mathrm{m} / \mathrm{s}$

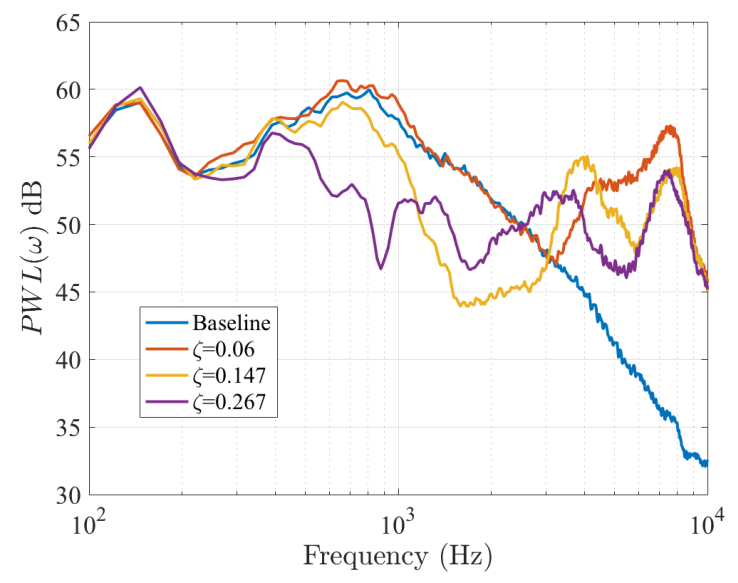

(b) $U=60 \mathrm{~m} / \mathrm{s}$

Fig. 10 Total sound power spectra for varying porous length $\zeta$ for porous open area $32.8 \%$ at two flow velocity $U$ of 40 and $60 \mathrm{~m} . \mathrm{s}$

We now consider the influence of porous open section length on noise reductions. Sound power reductions are plotted in Figure 11 against non-dimensional frequency $F \zeta L_{0} / U_{0}$ for various open section length of $\zeta=0.06$, 0.147, 0.267 respectively for $32.8 \%$ open area ratio and flow velocities of 40 and $60 \mathrm{~m} / \mathrm{s}$. Noise reductions of up to $12 \mathrm{~dB}$ are observed and the noise reductions are observed to be a function of open section length $\zeta$. This figure reveals a good collapse on the peak noise reduction frequency of $F \zeta L_{0} / U_{0}=0.55$ at both velocities. A second peak frequency around $F \zeta L_{0} / U_{0}=1.2$ is also observed for $\zeta=0.267$ where as for the other cases it may be masked by the self-noise increase. The reason for this frequency dependence is not clear and more work is required to explain this observation. A possible noise reduction mechanism might be due to destructive interference between different sources along the porous medium. The shift in peak frequency compared to analytic result of $F \zeta L_{0} / U_{0}=0.5,1.5$ can be due to change in effective convective speed or change in precise location of sources along the porous leading edge. However, more work is required to explain this hypothesis. More work is required to understand the effect of porosity and hole diameters on noise reductions.

\section{Discussion}

This section compares the theoretical and experimental results for the noise reductions possible by use of porous leading edges.

The theoretical model demonstrates the possibility of appreciable reductions in the far-field noise due to an incident gust, with the largest reductions at low to mid frequencies. The prominent role played by the diffracted fields arising from the sharp leading and trailing edge means the reduction is sensitive to the locations of the material junctions and the porosity of the leading edge. Experimentally it is found that the noise reductions collapse when plotted against Strouhal number

$$
f \zeta L_{0} / U_{0}
$$

where $\zeta L_{0}$ is the length of open porous section and $U_{0}$ is the mean background flow speed. The largest theoretical noise reduction for a porous leading edge affixed to a semi-infinite impermeable plate also occurs at a Strouhal number of $1 / 2$ (based upon the length of the open section) as is found experimentally. However, when modelling a finite plate this trend appears to be subsumed within oscillations in the spectra associated with the length scale associated finite chord, indicating that acoustic scattering interference introduced by the porous-rigid junction may not play a significant role in 


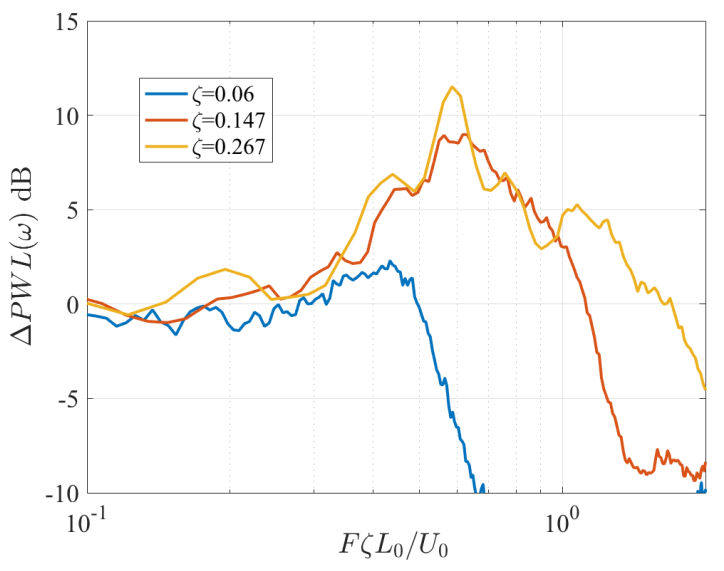

(a) $U=40 \mathrm{~m} / \mathrm{s}$

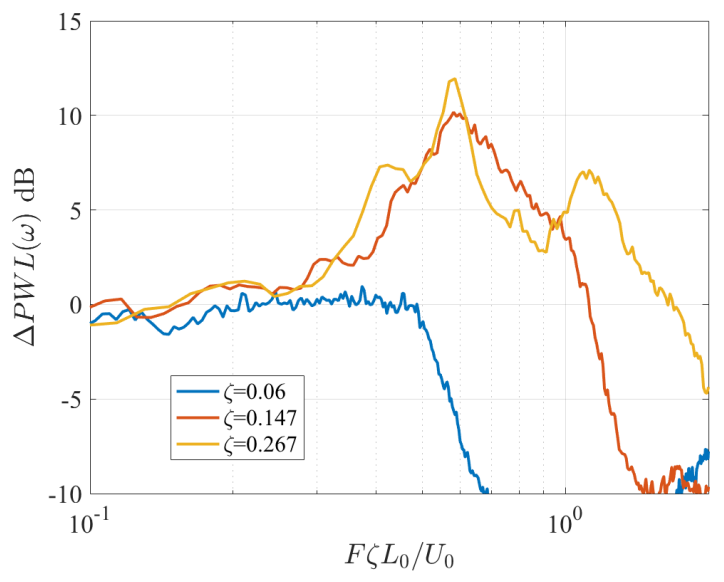

(b) $U=60 \mathrm{~m} / \mathrm{s}$

Fig. 11 Sound power reductions levels for varying porous length $\zeta$ for porous open area $32.8 \%$ at two flow velocities $U$ of 40 and $60 \mathrm{~m} . \mathrm{s}$

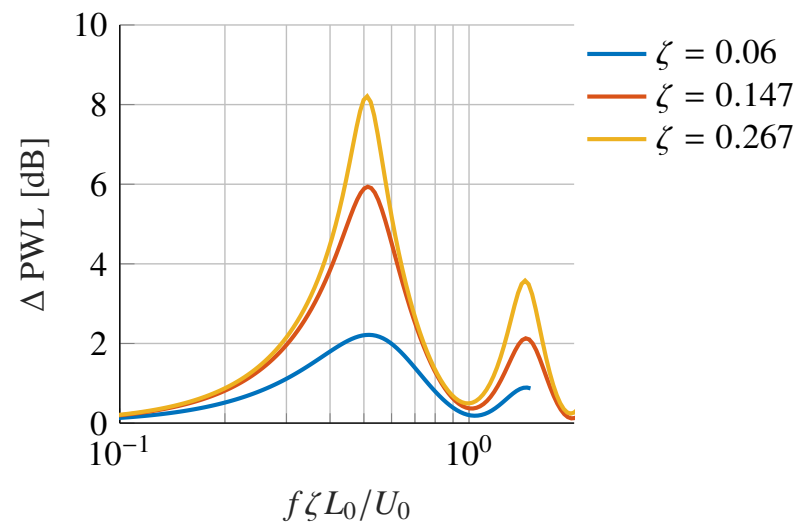

(a) $\mu=6$

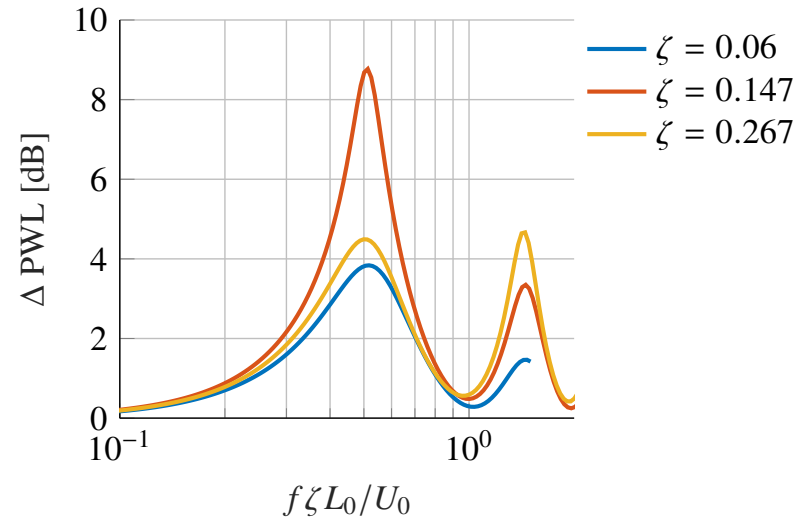

(c) $\mu=10$

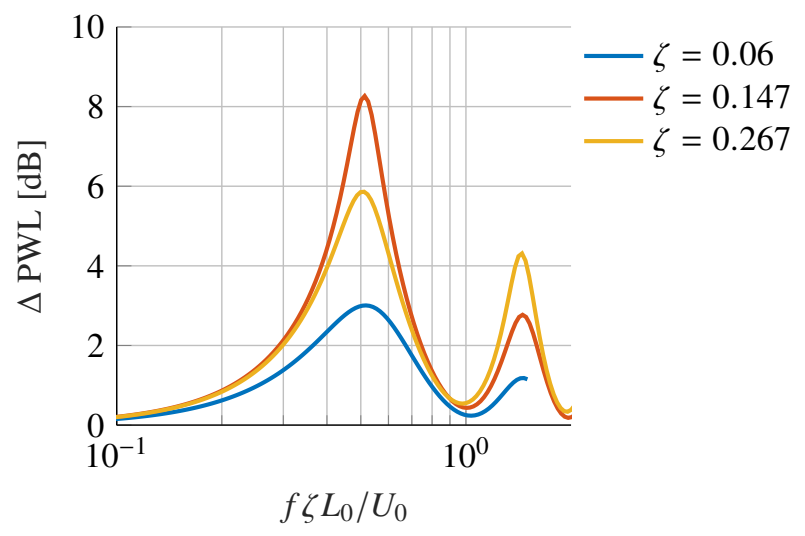

(b) $\mu=8$

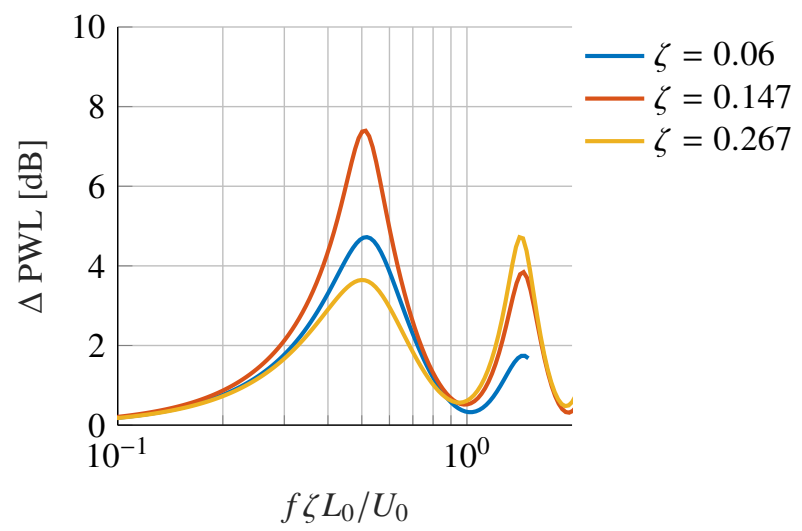

(d) $\mu=12$

Fig. $12 \triangle P W L$ for a semi-infinite impermeable rigid plate with a leading edge of length $\zeta L_{0}$ and porosity parameter $\mu=6,8,10,12$. For reference, using equation 17 the experimental case considered corresponds to $\mu \approx 10.5$ using a Rayleigh conductivity $K_{R}=2 R$ that does not model the effect of mean flow. 
the reductions observed in reality.

In figure 12 we present a typical reductions in PWL for a porous leading edge associated with values of $\mu$ similar to that determined by the experimental parameters considered using equation (17), that is $\mu \approx 10.5$. We observe broad qualitative agreement in the noise reductions found at low frequencies. The theoretical model does not predict the significant increase in noise at high frequencies, which is likely to be due to increased self-noise due to cross flow through the plate, and may mask reductions that would otherwise be observed. This increase in noise may be also associated with a breakdown of the theoretical model as the streamwise wavelength of the disturbance becomes comparable to the perforation diameter. As previously noted the largest reduction typically occurs at a Strouhal number of approximately $1 / 2$ as in the experimental results. The quantitative prediction is also of a similar order of magnitude to that found experimentally. For larger $\mu$ the reduction does not occur for the largest porous leading edge length; this is likely to be associated with changed interference between scattered fields from the porous leading edge and porous-rigid junction. The theoretical model is valid for small open fraction $\alpha_{H} \ll 1$ and small reduced frequency $k_{0} R \ll 1$. The experimental case considered has $\alpha_{H}=0.33$ has $f R / c_{0}<0.088$ for $f<10000 \mathrm{~Hz}$, each to 2 significant figures. Better agreement may be found for experimental results considering smaller open fractions and reduced frequencies.

We now investigate the sensitivity of noise reductions to flow speed. Figure 13 presents a side by side comparison of $\mathrm{PWL}$ reductions for varying for two velocities $40 \mathrm{~m} / \mathrm{s}$ in panel (a) and $60 \mathrm{~m} / \mathrm{s}$ in panel (b), for comparison with figure 11 Figure 14a shows the reduction in total noise versus non-dimensional frequency $\left(f \zeta L_{0} / U_{0}\right)$ at speeds of 20,40 and 60 $\mathrm{m} / \mathrm{s}$ for $32.8 \%$ open area porous leading edge with $\zeta=0.267$. The noise reduction spectra follows a non-dimensional frequency dependence $\left(f \zeta L_{0} / U_{0}\right)$ which is consistent with the analytical predictions as shown in figure $7 \mathrm{a}$ and described on page 10 . As in the experimental results, the theoretical results are broadly unchanged for the cases considered; we note only that the peak reduction $\zeta=0.147$ slightly larger for the velocity of $40 \mathrm{~m} / \mathrm{s}$ this trend as discussed above.

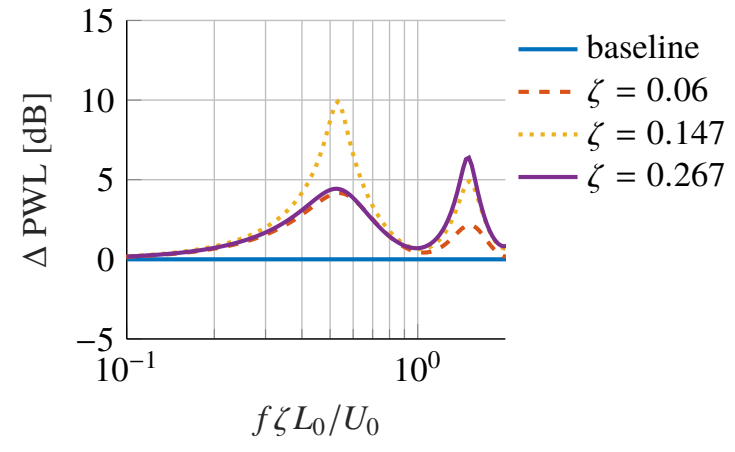

(a) $U_{0}=40 \mathbf{m} / \mathbf{s}$

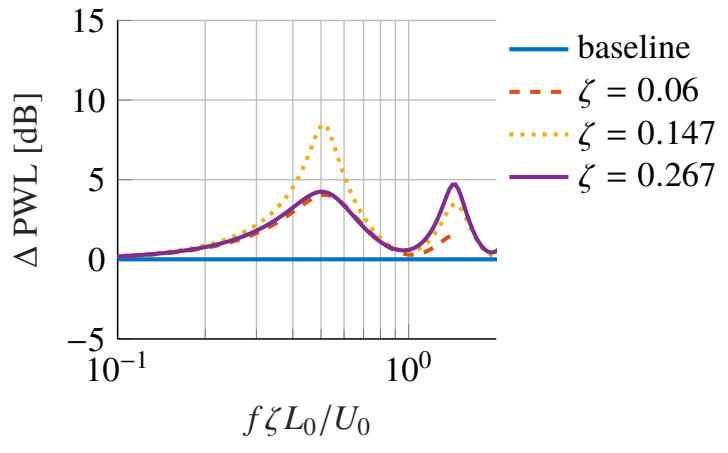

(b) $U_{0}=60 \mathbf{m} / \mathbf{s}$

Fig. $13 \quad \triangle$ PWL for a semi-infinite impermeable rigid plate with a leading edge of length $\zeta$ for two mean flow velocities $U_{0}$, using $\mu=10.5$ as computed from the experimental parameters and equation 17

In figure 14 we compare the theoretical and experimental reductions in PWL for a porous section of length $0.267 L_{0}$, varying the velocity. Panel (a) shows the experimental results, and panel (b) shows the theoretical prediction for $\mu=6.5$. Figure 15 presents results for two velocities overlayed. We observe a qualitative agreement in two peak reduction zones around a Strouhal number of approximately 0.5 to 0.55 and 1.2 (experiment) to 1.5 (theory). Further work is required to understand this offset; it may be affected by considering a frequency dependent porosity parameter $\mu$, or due to a change in effective convective speed or change in the precise location of sources along the porous leading edge. At best we observe agreement in magnitude of these reductions to $2 \mathrm{~dB}$ to $4 \mathrm{~dB}$, which considering the simplicity of the current model for the porous boundary condition may be promising. Panel (b) demonstrates that considering a porosity parameter or $\mu=6.5$, smaller than that given by equation (17), can give reasonable qualitative agreement with the experimental results; further work is required to consider a more appropriate model for the porosity value.

Future work could investigate more sophisticated porosity models, such as those developed in [9] and further interrogate the model with a broader experimental study. The value of such work would be to isolate the acoustic scattering and absorption effects from alternative mechanisms that may be present; for instance modifications to the incident disturbance, as a thicker boundary layer displacing the turbulence further from the surface effectively increasing leading edge thickness to reduced gust interaction noise as described in [5]. 


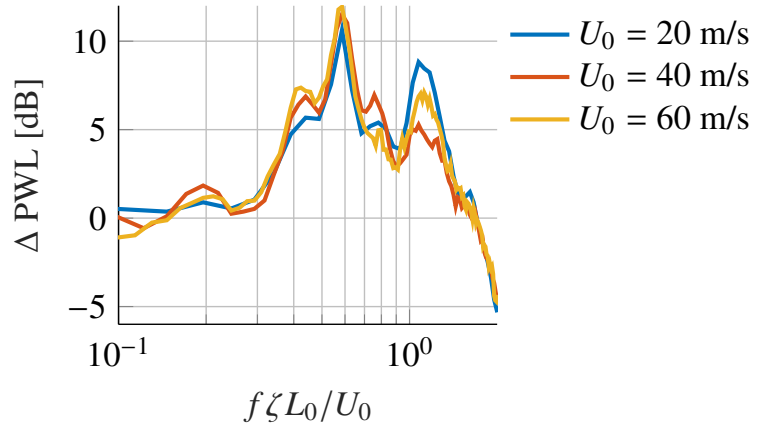

(a) Experimental results for $32.8 \%$ open area

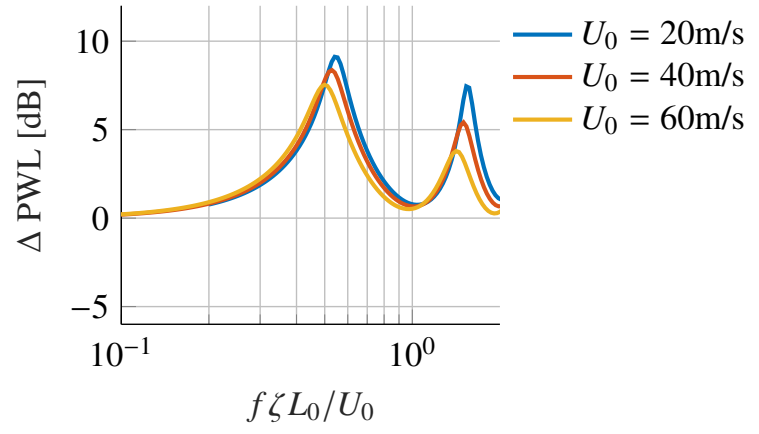

(b) Analytic results for $\mu=6.5$

Fig. 14 Comparison between theoretical and experimental PWL reductions for a leading edge of length $\zeta=0.267 L_{0}$, varying velocity. Experimental results for $\Delta \mathbf{P W L}$ are presented in (a). In (b) theoretical results are presented for a semi-infinite impermeable rigid plate for $\mu=6.5$, which gives reasonable fit to experimental results. A value of $\mu=10.5$ would be in accordance with the experimental parameters and equation (17), but note that such a computation of the porosity parameter $\mu$ neglects the effects of mean flow.

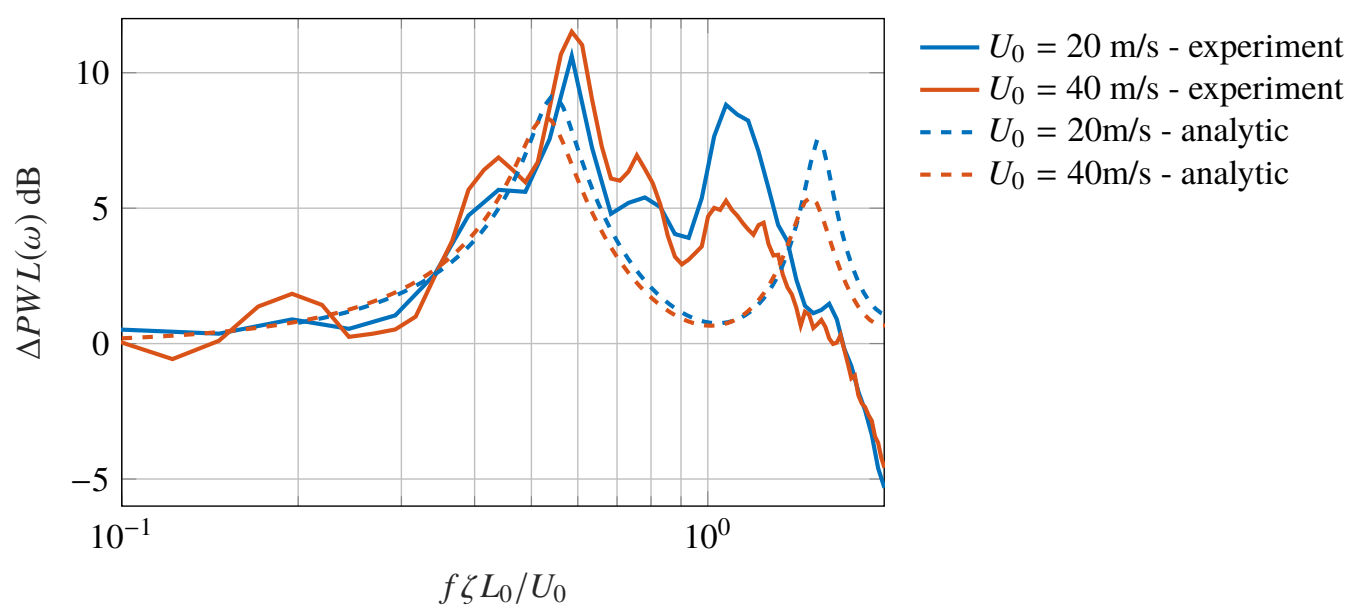

Fig. 15 Comparison of model and experiment: $\triangle$ PWL comparison varying $U$ for $32.8 \%$ open area porous leading edge with $\zeta=0.267, \mu=6.5$ 


\section{Conclusion}

This paper presents a simple acoustic scattering model for a flat plate with a porous leading edge, together with experimental results for a noise generation by a flat plate with a perforated leading edge section. The theoretical scattering model applies an iterative approach to matrix Wiener-Hopf problems to investigate gust-aerofoil interaction for porous leading edges, accounting for the acoustic impact of an acoustically softer leading edge, and scattering from the additional material junction. We obtain quantitative results for the noise reductions obtained by increasing the porosity parameter $\mu$ and length of porous extension $\zeta$. Overall qualitative agreement is found between the experimental results and a theoretical model for a semi-infinite flat plate with a porous leading edge at low frequencies, with the maximum noise reduction typically found at a Strouhal number of approximately $1 / 2$ based upon the length of the leading edge and mean flow velocity. Moreover, the quantitative values predicted are of the similar order of magnitude to those found experimentally. Further work incorporating a more sophisticated boundary condition for the porous section and considering a larger range of experimental test cases should clarify the validity of the theoretical model. This will provide a solid platform for understanding the role of acoustic scattering in basic porous leading edge designs, and so help inform more sophisticated implementations.

\section{Acknowledgments}

M. J. Priddin thanks A. V. Kisil for helpful discussions regarding the iterative Wiener-Hopf method, and thanks S. Olver for advice regarding the use of SingularIntegralEquations.jl. L. J. Ayton acknowledges support from EPSRC Early Career Fellowship EP/P015980/1.

\section{References}

[1] Geyer, T., Sarradj, E., and Fritzsche, C., "Measurement of the noise generation at the trailing edge of porous airfoils," Experiments in Fluids, Vol. 48, No. 2, 2010, pp. 291-308. doi:10.1007/s00348-009-0739-x.

[2] Geyer, T., "Trailing edge noise generation of porous airfoils," Ph.D. thesis, Brandenburg University of Technology Cottbus, 2011.

[3] Geyer, T. F., and Sarradj, E., "Trailing edge noise of partially porous airfoils," 20th AIAA/CEAS Aeroacoustics Conference, 2014, p. 3039.

[4] Roger, M., Schram, C., and De Santana, L., "Reduction of Airfoil Turbulence-Impingement Noise by Means of Leading-Edge Serrations and/or Porous Material," 19th AIAA/CEAS Aeroacoustics Conference, Aeroacoustics Conferences, American Institute of Aeronautics and Astronautics, 2013. doi:10.2514/6.2013-2108.

[5] Geyer, T. F., Lucius, A., Schrödter, M., Schneider, M., and Sarradj, E., "Reduction of Turbulence Interaction Noise Through Airfoils With Perforated Leading Edges," Acta Acustica united with Acustica, Vol. 105, No. 1, 2019, pp. $109-122$. doi:info:doi/10.3813/AAA.919292.

[6] Lee, S., "Reduction of blade-vortex interaction noise through porous leading edge," AIAA Journal, Vol. 32, No. 3, 1994, pp. 480-488. doi:10/dvs9qr.

[7] Avallone, F., Casalino, D., and Ragni, D., "Impingement of a propeller-slipstream on a leading edge with a flow-permeable insert: A computational aeroacoustic study," International Journal of Aeroacoustics, 2018, p. 1475472X18788961. doi:10/gd8c3v.

[8] Jaworski, J. W., and Peake, N., "Aerodynamic noise from a poroelastic edge with implications for the silent flight of owls," Journal of Fluid Mechanics, Vol. 723, 2013, pp. 456-479. doi:10/f4vkds.

[9] Howe, M. S., Acoustics of Fluid-Structure Interactions, Cambridge University Press, Cambridge, 1998. doi:10.1017/ CBO9780511662898.

[10] Ayton, L. J., “Acoustic scattering by a finite rigid plate with a poroelastic extension,” Journal of Fluid Mechanics, Vol. 791, 2016, pp. 414-438. doi:10/f8bvg3.

[11] Kisil, A., and Ayton, L. J., "Aerodynamic noise from rigid trailing edges with finite porous extensions," Journal of Fluid Mechanics, Vol. 836, 2018, pp. 117-144. doi:10/gcpv8k.

[12] Cavalieri, A. V., Wolf, W. R., and Jaworski, J., "Acoustic scattering by finite poroelastic plates," 20th AIAA/CEAS Aeroacoustics Conference, 2014, p. 2459. 
[13] Cavalieri, A. V. G., Wolf, W. R., and Jaworski, J. W., "Numerical solution of acoustic scattering by finite perforated elastic plates," Proc. R. Soc. A, Vol. 472, No. 2188, 2016, p. 20150767. doi:10.1098/rspa.2015.0767.

[14] Hajian, R., and Jaworski, J. W., "The steady aerodynamics of aerofoils with porosity gradients," Proceedings of the Royal Society A: Mathematical, Physical and Engineering Science, Vol. 473, No. 2205, 2017, p. 20170266. doi:10/gb2663.

[15] Abrahams, I. D., “The application of Pade approximants to Wiener-Hopf factorization,” IMA Journal of Applied Mathematics, Vol. 65, No. 3, 2000, pp. 257-281. doi:10/fvj5fk.

[16] Kisil, A. V., "An Iterative Wiener-Hopf Method for Triangular Matrix Functions with Exponential Factors," SIAM Journal on Applied Mathematics, Vol. 78, No. 1, 2018, pp. 45-62. doi:10/gcp3w2.

[17] Slevinsky, R. M., and Olver, S., "A fast and well-conditioned spectral method for singular integral equations," Journal of Computational Physics, Vol. 332, 2017, pp. 290-315. doi:10/f9qr27.

[18] Crighton, D. G., and Leppington, F. G., "Scattering of aerodynamic noise by a semi-infinite compliant plate," Journal of Fluid Mechanics, Vol. 43, No. 04, 1970, p. 721. doi:10.1017/S0022112070002690.

[19] Glegg, S., and Devenport, W., Aeroacoustics of Low Mach Number Flows: Fundamentals, Analysis, and Measurement, Academic Press, 2017.

[20] Chong, T. P., Joseph, P. F., and Davies, P. O. A. L., "A Parametric Study of Passive Flow Control for a Short, High Area Ratio 90 deg Curved Diffuser," Journal of Fluids Engineering, Vol. 130, No. 11, 2008, p. 111104. doi:10/bpv6zw.

[21] Narayanan, S., Chaitanya, P., Haeri, S., Joseph, P., Kim, J. W., and Polacsek, C., "Airfoil noise reductions through leading edge serrations," Physics of Fluids, Vol. 27, No. 2, 2015, p. 025109. doi:10/f637xx.

[22] Ayton, L. J., and Chaitanya, P., "An analytical and experimental investigation of aerofoil-turbulence interaction noise for plates with spanwise-varying leading edges,” Journal of Fluid Mechanics, Vol. 865, 2019, p. 137-168. doi:10.1017/jfm.2019.78. 